\title{
A formação conceitual sob a perspectiva teórica de Vygotsky, Leontiev e Galperin
}

\author{
Conceptual formation from the theoretical perspective of Vygotsky, Leontiev and Galperin \\ Formación conceptual desde la perspectiva teórica de Vygotsky, Leontiev y Galperin
}

Recebido: 24/03/2021 | Revisado: 30/03/2021 | Aceito: 11/04/2021 | Publicado: 21/04/2021

\author{
Rannyelly Rodrigues de Oliveira \\ ORCID: https://orcid.org/0000-0002-3850-5237 \\ Universidade Federal do Rio Grande do Norte, Brasil \\ E-mail: ranny.math.06@gmail.com \\ Ana Paula Bispo da Silva \\ ORCID: https://orcid.org/0000-0001-8465-0614 \\ Universidade Estadual da Paraíba, Brasil \\ E-mail: silva.anapaulabispo@gmail.com
}

\begin{abstract}
Resumo
Este trabalho apresenta uma resenha do livro: "Vygotsky, Leontiev e Galperin: formação de conceitos e princípios didáticos". O livro foi escrito por Isauro Beltrán Núñez e abrange a formação de conceitos na perspectiva teórica de Vygotsky, Leontiev e Galperin, apresentando uma descrição dos princípios didáticos assumidos a partir da análise da Teoria Histórico-Cultural, Teoria da Atividade e Teoria da Assimilação. Para isso, foi apresentado o itinerário formativo das teorias propostas por Vygotsky, Leontiev e Galperin destacando os aspectos em que elas se complementam e divergem. Portanto, o autor evidenciou a relevância do planejamento didático com enfoque na Teoria da Assimilação em articulação com as etapas da Atividade Histórico-Cultural para a prática docente e aprendizagem. Por fim, buscou-se instigar o leitor a refletir na possibilidade de articular as concepções de Galperin com outras teorias socioculturais como, por exemplo, a Teoria da Objetivação de Luis Radford, a qual tem muitas implicações no ensino de Ciências e Matemática vislumbrando, dessa maneira, sua extensão a outras áreas de conhecimento.
\end{abstract}

Palavras-chave: Teoria histórico-cultural; Teoria da atividade; Teoria da assimilação.

\begin{abstract}
This work presents a review of the book: "Vygotsky, Leontiev and Galperin: formation of didactic concepts and principles". The book was written by Isauro Beltrán Núñez and covers the formation of concepts in the theoretical perspective of Vygotsky, Leontiev and Galperin, presenting a description of the didactic principles assumed from the analysis of Historical-Cultural Theory, Activity Theory and Theory of Assimilation. For this, the formative itinerary of the theories proposed by Vygotsky, Leontiev and Galperin was presented, highlighting the aspects in which they complement and diverge. Therefore, the author highlighted the relevance of didactic planning with a focus on Assimilation Theory in conjunction with the stages of Historical-Cultural Activity for teaching and learning practice. Finally, we sought to encourage the reader to reflect on the possibility of articulating Galperin's conceptions with other sociocultural theories, such as, for example, the Theory of Objectification by Luis Radford, which has many implications for the teaching of Science and Mathematics, with a view way, its extension to other areas of knowledge.
\end{abstract}

Keywords: Historical-cultural theory; Activity theory; Assimilation theory.

\section{Resumen}

Este trabajo presenta una revisión del libro: "Vygotsky, Leontiev y Galperin: formación de conceptos y principios didácticos". El libro fue escrito por Isauro Beltrán Núñez y cubre la formación de conceptos en la perspectiva teórica de Vygotsky, Leontiev y Galperin, presentando una descripción de los principios didácticos asumidos a partir del análisis de la Teoría Histórico-Cultural, Teoría de la Actividad y Teoría de la Asimilación. Para ello, se presentó el itinerario formativo de las teorías propuestas por Vygotsky, Leontiev y Galperin, destacando los aspectos en los que se complementan y divergen. Por ello, el autor resaltó la relevancia de la planificación didáctica con enfoque en la Teoría de la Asimilación en conjunto con las etapas de Actividad Histórico-Cultural para la práctica de la enseñanza y el aprendizaje. Finalmente, se buscó animar al lector a reflexionar sobre la posibilidad de articular las concepciones de Galperin con otras teorías socioculturales, como por ejemplo, la Teoría de la Objetificación de Luis Radford, que tiene muchas implicaciones para la enseñanza de las Ciencias y las Matemáticas, con un modo de vista, su extensión a otras áreas del conocimiento.

Palabras clave: Teoría histórico-cultural; Teoría de la actividad; Teoría de la asimilación. 


\section{Introdução}

Este trabalho apresenta uma discussão, na forma de resenha, sobre o livro, da autoria de Isauro Beltrán Núñez, intitulado de "Vygotsky, Leontiev e Galperin: formação de conceitos e princípios didáticos" que aborda a formação de conceitos na perspectiva teórica de Vygotsky, Leontiev e Galperin, apresentando uma descrição dos princípios didáticos assumidos a partir da análise da teoria histórico-cultural, teoria da atividade e teoria da assimilação. Ademais, Núñez possui graduação e mestrado em Química e doutorado em Ciências Pedagógicas, atualmente, é professor titular do Centro de Educação da Universidade Federal do Rio Grande do Norte atuando como bolsista de Produtividade em Pesquisa pelo Conselho Nacional de Desenvolvimento Científico e Tecnológico - CNPq.

\section{Aporte Teórico}

Neste tópico, serão apresentadas as ideias principais que sustentam o aporte teórico deste trabalho. Iniciando pelas concepções de Vygotsky, pode-se descrever que sua teoria histórico-cultural é oriunda de um contexto revolucionário, onde se preconizava a concepção de um novo método científico e de uma ciência humana. Esse cenário foi marcado pela primeira guerra mundial em que se observava a transição do czarismo para o comunismo. A teoria de Vygotsky desenvolveu-se através de um processo dialético na busca da construção de um novo homem, uma nova sociedade e uma educação inovadora. (Bortolanza \& Ringel, 2016, p. 1039).

Os elementos de ordem social, histórica e cultural compõem a espinha dorsal da teoria de Vygotsky, na qual se estuda como o pensamento e a linguagem estão relacionados entre si e com a prática social, como se dá a formação conceitual científica, e como se efetiva a tríade: trabalho - educação - práticas educativas. O escopo neoliberal e pós-moderno da teoria de Vygotsky ignora a perspectiva teórica marxista, a qual defende o trabalho como princípio essencial do "ser social". Além disso, essa vertente teórica compreende o docente como um ator coadjuvante no processo de apropriação de uma cultura universal. (Cerezuela \& Mori, 2015, p. 1256).

No âmbito educacional, Vygotsky afirma que a aprendizagem e o desenvolvimento são dois conceitos diferentes. O processo de aprendizagem direciona o desenvolvimento. Ou seja, a aprendizagem em si é um conjunto de práticas que visam alcançar a "zona de desenvolvimento proximal” do aluno. Para Vygotsky, a aprendizagem e o desenvolvimento são inerentemente sociais, de maneira que o desenvolvimento não ocorre apenas internamente no indivíduo, mas que parte, primeiramente, das relações (externas) sociais. (Lobman, 2016, p. 133).

Na perspectiva de transformação social, a Teoria da Atividade foi desenvolvida, principalmente, por Leontiev. Santos e Asbahr (2020, p. 4) fazem uma análise das ideias principais dessa teoria a partir da obra de Leontiev: Actividad, conciencia y personalidad. Os autores evidenciam o conceito de atividade como sendo uma unidade vital que orienta o sujeito no mundo objetivo. Assim sendo, para Leontiev:

A atividade é uma unidade molecular, não uma unidade aditiva da vida do sujeito corporal, material. Em um sentido mais estrito, quer dizer, a nível psicológico, é a unidade da vida mediada pelo reflexo psicológico, cuja função real consiste em que orienta ao sujeito no mundo objetivo. Em outras palavras, a atividade não é uma reação nem um conjunto de reações, mas um sistema que tem estrutura, suas transições e transformações internas, seu desenvolvimento (Leóntiev, 1978, pp. 66-67).

O conceito de atividade também se expressa em sentidos diferentes. Para Vygotsky, a atividade é compreendida como uma unidade norteada por signos e ferramentas, enquanto Leontiev entende a atividade como um conceito coletivo que age como base da consciência humana. Leontiev concentrou-se no estudo da atividade e no objeto dessa atividade. O objeto pode 
ser real ou ideal. A necessidade de algo define o objeto. A necessidade é o motivo real da atividade e é ela que permite reconhecer o objeto da atividade. (Cenci \& Damiani, 2018, p. 933).

A teoria de Galperin fundamenta-se no princípio de que é possível realizar, através de uma atividade planejada, processos mentais que atuem como órgãos funcionais da atividade. Ou seja, uma atividade planejada pode oportunizar a aprendizagem de conceitos escolares e o desenvolvimento de habilidades. Galperin desenvolveu o princípio de interiorização criado por Vygotsky e o princípio da atividade de Leontiev, contudo, conduzindo-os para o entendimento do funcionamento dos aspectos psicológicos superiores. O cerne da teoria de Galperin se firma em acreditar que as novas ações mentais, são de natureza comportamentalmente objetiva, que são impulsionadas, a priori, com aporte em objetos externos e devido à sua manipulação, as ações se mobilizam na dimensão mental e constituem-se como propriedades da psique. (Núñez \& Ramalho, 2015, p. 8).

Doravante, será apresentada a estrutura metodológica deste trabalho, a qual foi organizada conforme os capítulos do livro, a fim de situar melhor o leitor na discussão. Algumas obras do autor do livro que versam sobre as perspectivas teóricas de Vygotsky, Leontiev e Galperin o conduziram a escrever o livro. Assim, na composição do livro, o qual é tratado nesta resenha, destacam-se as seguintes referências: Núñez e González (1997, 1998), Núñez (1996), Núñez e Farias (2004).

\section{Estrutura Metodológica}

Este artigo trata de uma resenha de um livro. Assim sendo, este trabalho caracteriza-se como uma pesquisa bibliográfica cujo objetivo é fazer uma análise teórica das ideias propostas por Vygotsky, Leontiev e Galperin pertinentes à formação de conceitos numa perspectiva didática. Vale compreender que, segundo Mascarenhas (2012, p. 49), essa tipologia de pesquisa é centrada no estudo de obras, livros e artigos científicos. Cervo, Bervian e Silva (2007, p.61) relatam que esse estudo bibliográfico assume uma estrutura descritiva vislumbrando sua análise. Dessa forma, acentua-se que:

A pesquisa descritiva observa, registra, analisa e correlaciona fatos ou fenômenos (varáveis) sem manipulá-los [...] busca conhecer as diversas situações e relações que ocorrem na vida social, política, econômica e demais aspectos do comportamento humano, tanto do indivíduo tomado isoladamente como de grupos e comunidades mais complexas (Cervo, Bervian \& Silva, 2007, p. 61).

O livro é organizado em cinco capítulos. No capítulo 1, o autor explica que Vygotsky (1896-1934) foi quem desenvolveu a Teoria Histórico-Cultural, na qual se discute a relação entre os processos de aprendizagem e desenvolvimento, evidenciando as suas concepções de aprendizagem associadas aos mecanismos de pensamento e linguagem. No capítulo 2, a Atividade é discutida segundo as concepções de Leontiev (1903-1979), o qual desenvolveu a Teoria da Atividade numa vertente da psicologia soviética, realizando uma análise crítica da Teoria Histórico-Cultural de Vygotsky através da sua argumentação de que não são os conceitos, mas sim a atividade real o que liga o sujeito a sua realidade, o que designa o desenvolvimento da consciência.

No capítulo 3, são discutidas as concepções de Galperin que complementam as ideias de Leontiev e de Vygotsky. Nesse capítulo, pode-se observar que o autor pontua que as teorias de Leontiev e de Vygotsky não explicam como ocorre o processo de internalização da atividade externa em atividade interna. No capítulo 4, o autor descreve os princípios didáticos, assumidos de acordo com as perspectivas teóricas de Leontiev, Vygotsky e Galperin. Esses princípios são gerados a partir das regularidades que gerenciam o ensino. Esses princípios evidenciam que o ensino deve ter um caráter educativo, científico, consciente e objetal. Por fim, o capítulo 5 dá continuidade às concepções de Galperin, através de uma discussão sobre as transformações que ocorrem no planejamento didático do processo de formação de habilidades no ensino. 


\section{Resultados e Discussões}

No capítulo 1, é explicado que a Teoria Histórico-Cultural vai contra a concepção, da psicologia ocidental tradicional, sobre o conceito de homem, educação e aprendizagem. Pois, para Vygotsky, a aprendizagem não é apenas uma realização individual, mas sim uma atividade de abrangência social. Desse modo, a aprendizagem abrange três aspectos essenciais: o caráter social, a atividade e a mediação.

A perspectiva teórica de Vygotsky atribui ao estudante uma protagonização no processo de aprendizagem, de maneira que ele se comporte como indivíduo ativo e consciente, que está sendo conduzido por intencionalidades pedagógicas. A aprendizagem resulta da interação entre professores e alunos e da forma como esse grupo age sobre determinados objetos condicionados por fatores sócio-históricos. Assim, a aprendizagem é compreendida como uma atividade humana que assume um caráter social, gerando mudanças psíquicas e/ou físicas tanto no estudante como no objeto da atividade.

Nesse sentido, a aprendizagem, com ênfase na atividade, depende da interação das pessoas. A fase inicial da atividade é interpsicológica, à medida que ocorre a internalização da assimilação, a atividade passa a se manifestar no plano intrapsicológico. Segundo Vygotsky, a determinação social do pensamento se justifica não apenas por suas transformações quantitativas, mas, também nas suas mudanças qualitativas. Essa atividade (entre sujeito e objeto) transformadora é mediada por ferramentas, as quais são denominadas por Vygotsky de signos e de objetos materiais ou espirituais.

A aprendizagem pode proporcionar o desenvolvimento do sujeito, a partir da transformação qualitativa de suas estruturas psicológicas elementares em funções psicológicas superiores. O funcionamento psicológico do ser humano tem teor cultural e, consequentemente, torna-se histórico. Durante o desenvolvimento de uma sociedade, são elaborados conteúdos culturais que atuam como mediadores na interação realizada entre as pessoas e o mundo. Esse conteúdo histórico-cultural é o objeto assimilado na socialização e no desenvolvimento da personalidade de um indivíduo. Dessa maneira, é relevante compreender que existem dois níveis de desenvolvimento: um está associado às capacidades e potencialidades reais e o outro às possibilidades de aprendizado com ajuda dos colegas. A diferença entre esses dois níveis é denominada por Vygotsky de Zona de Desenvolvimento Proximal.

Ainda no primeiro capítulo, o autor discute a formação de conceitos fundamentando-se na teoria de Vygotsky, o qual ao analisar as crianças, acreditava que o desenvolvimento do seu pensamento verbal estava associado à sua formação conceitual, que ocorre sob duas perspectivas: uma vinculada ao modo de pensamento espontâneo desenvolvido pela criança nas suas vivências diárias e a outra ao que se desenvolve no âmbito escolar. A sociogênese da formação conceitual espontânea, não ocorrendo exclusivamente nos estágios de maturação biológica, acontece em três fases do pensamento: pensamento sincrético, pensamento por complexos e pensamento propriamente conceitual. No pensamento sincrético, é onde os significados de uma palavra são internalizados. O pensamento por complexos abrange as relações concretas e factuais entre os aspectos discretos da experiência. E, a terceira fase, é um produto obtido de uma ação real e complexa do pensamento, envolvendo operações (mais elaboradas) mentais analíticas e sintéticas.

Ademais, a formação de um conceito científico acontece a partir da incorporação de novos significados e no desenvolvimento das interrelações entre eles quando abordados em uma teoria científica. A aprendizagem dos conteúdos (conceitos) escolares permite o aluno a construir saberes que não fazem parte de suas experiências sensíveis e concretas do diaa-dia. Dessa forma, as redes conceituais se reorganizam em teorias pelas quais os indivíduos se dispõem para interpretar e mudar a realidade no contexto social. Os conceitos devem ser entendidos a partir da interação entre sentidos e significados que eles assumem na delimitação entre conceitos espontâneos e conceitos científicos oriundos do conteúdo escolar. Isto posto, para Vygotsky, o paradigma pelo qual se deve avaliar as transformações na atividade mental do processamento conceitual são: aspectos quantitativos e qualitativos da generalização conceitual, grau de abstração e de incorporação dos conceitos ao sistema de redes conceituais. 
No capítulo 2, o autor versa sobre a atividade segundo a perspectiva teórica de Leontiev pertinente à Teoria da Atividade. Tendo em vista que a comunicação é obtida na atividade prática, assim, Leontiev valorizava mais a atividade prática dos indivíduos e de suas relações com o mundo do que a comunicação. Nesse sentido, a Teoria da Atividade aborda os processos de assimilação de conceitos científicos na aprendizagem do conteúdo escolar, desse modo, integra dialeticamente as ideias de Vygotsky. Doravante, esse escopo teórico se complementa com as acepções de Galperin, o qual desenvolveu a teoria da assimilação por etapas de ações mentais.

Para Leontiev, a atividade humana é um processo mediador que permite a interação entre o ser humano (sujeito) e a realidade (objeto) a ser transformada por ele. Nessa dialética, tanto o objeto como o sujeito são transformados proporcionando, assim, mudanças na personalidade do homem. A atividade é intrinsecamente humana, ou seja, é por meio da atividade prática que o ser humano age sobre a natureza, a sociedade e sobre si mesmo. A atividade é a forma elementar, socionormativa e assimilada da natureza humana. Assim, a assimilação das vivências acumuladas pelo sujeito não constitui apenas um processo adaptativo ao meio, mas também um processo ativo de transformação.

Segundo o viés da teoria de Leontiev, há diversas tipologias de atividades, como por exemplo: atividade prática, gnosiológica, valorativa, comunicativa, dentre outras. Segundo o autor, essas devem ser as atividades desenvolvidas juntamente com os alunos durante a aprendizagem. É valido compreender que, assumindo o pressuposto de que ensinar é um processo organizacional da atividade cognitiva dos alunos, quando se realiza atividades valorativas (formação de valores) e de comunicação (socialização), é possível alcançar o desenvolvimento integral da personalidade dos estudantes.

Com isso, pode-se entender que a Teoria Histórico-Cultural e a Teoria da Atividade não podem ser estudadas à borda do conceito de personalidade. $\mathrm{O}$ que aconteceu nas divulgações ocidentais. $\mathrm{O}$ desenvolvimento integral da personalidade é uma premissa fundamental abordada nos trabalhos de Vygotsky e Leontiev, de maneira que suas concepções estão bastante associadas à noção de personalidade oriunda do materialismo dialético-histórico, em que a compreende como um sistema dinâmico de relações entre humanos, que são unidos por elos mútuos que possuem sempre, e em qualquer lugar, um caráter sócio-histórico.

Numa perspectiva do materialismo dialético-histórico, o autor explica que Leontiev buscou superar as colocações teóricas de Vygotsky e, dessa forma, enfatiza a atividade no estudo da formação das funções psicológicas superiores. As considerações teóricas formuladas por Leontiev têm implicações significativas no aprendizado de conceitos científicos dentro de ambientes escolares. Pois, a formação conceitual realizada na escola caracteriza uma atividade social, além de acontecer de forma mediada e contextualizada. Logo, compreende-se que apropriar-se de conceitos significa apoderar-se dos diversos tipos de atividades, nas quais os conceitos atuam como diretrizes que conduzem o estudante para o desenvolvimento integral de sua personalidade.

No capítulo 3, o autor defende que as concepções de Galperin complementam as ideias de Leontiev e de Vygotsky, pontuando que as teorias de Leontiev e de Vygotsky não explicam como ocorre o processo de internalização da atividade externa em atividade interna. A assimilação de conceitos abstratos não é o último estágio da construção do conhecimento, pois a sua aplicação na resolução de atividades é extremamente necessária para a transição da dimensão abstrata para a concreta, assim como, da concreta para abstrata.

Dessa forma, a mobilidade e a função ativa dos conceitos contribuem para o desenvolvimento das funções psicológicas superiores e da personalidade integral do ser humano. À vista disso, para adquirir novos conceitos, novas generalizações e novas habilidades, o estudante deve assimilar ações mentais compatíveis para tal situação, de maneira que essas ações sejam organizadas ativamente. Inicialmente, as ações são externas e, posteriormente, são transformadas em ações mentais internas. O que ficou conhecido como internalização, processo estudado por Galperin (1902-1988). 
Galperin desenvolveu uma pesquisa sobre os estágios de formação da atividade interna fundamentada na atividade externa, evidenciando as situações funcionais da atividade-orientação. Em seu trabalho, ele explorou o gerenciamento das transformações das ações, à medida que ele é generalizado e automatizado, assumindo um caráter particularmente psíquico. A teoria de Galperin tem implicação no processo de ensino, ao descrever que a assimilação do conhecimento é realizada em estágios fundamentais de sua formação, no que diz respeito a transição do contexto da vivência social para o da experiência individual.

De acordo com a Teoria de Galperin, o processo de internalização ocorre nas seguintes fases: $i$ ) fase motivacional, ii) determinação de uma base orientadora da ação, iii) formação da ação na dimensão material (materialização), iv) formação da ação na linguagem externa e $v$ ) ação na estrutura mental. Essas fases estão articuladas com as situações funcionais da ação: orientação, execução e controle. E, essas ações estão fortemente associadas à formação e aplicabilidade dos conceitos em seus limites de generalização.

Isso possibilita entender que o processo de formação conceitual deve ser organizado de maneira a considerar a atividade na qual se pretende formar um conceito; ou seja, em que o estudante deve assimilar o conceito; compreendendo os aspectos qualitativos que permitem o reconhecimento das etapas de formação conceitual. Destarte, a Teoria de Galperin efetiva-se na determinação de uma ação; concepção da materialização da ação e transformação da ação externa em interna. Com isso, a aprendizagem ocorre por assimilação dos conteúdos escolares através da atividade.

O capítulo 4 aborda a descrição dos princípios didáticos assumidos de acordo com os escopos teóricos de Leontiev, Vygotsky e Galperin. Esses princípios são concebidos a partir das regularidades que gerenciam o ensino. Esses princípios destacam que o ensino deve ter um caráter educativo, científico, consciente e objetal. Particularmente ao enfoque teórico de Galperin, pode-se destacar alguns princípios didáticos descritos a seguir.

Princípio do caráter seletivo da percepção: explica que uma informação chega ao aluno através dos órgãos sensoriais que produz a percepção, conduzindo o aluno a refletir sobre determinado objeto, porém, a reflexão não acontece de forma automática e sim seletiva, ou seja, o aluno seleciona sobre o que refletir. Princípio do caráter ativo da assimilação: significa que o estudante deve interagir com o objeto e agir sobre ele de forma consciente. Princípio da vinculação da aprendizagem com a vida: retrata que a atividade prática deve proporcionar a aplicação e validação da relevância social dos conteúdos escolares.

Princípio da ilustratividade e a materialização: a ilustratividade está associada à representação externa e a materialização é a fase concreta, assim, esse princípio abrange a organização de um conjunto de características criteriais do objeto. Princípio da unidade entre o ilustrativo e o verbal: é a transição do plano ilustrativo para o plano verbal. Princípio da retroalimentação: permite compreender a aprendizagem como um processo contínuo. Princípio do caráter sistêmico do objeto da assimilação: o conteúdo deve ser estruturado como núcleos invariantes de conhecimentos (essenciais e gerais) baseados em métodos. Princípio da sistematização do ensino: está relacionado aos conteúdos, os quais são organizados através de um número determinado de tarefas. Princípio da aprendizagem criativa: designa a realização de atividades que possam desenvolver a criatividade do estudante como característica de uma personalidade integral.

Em último momento, ainda sobre as concepções de Galperin, o capítulo 5 apresenta uma discussão sobre as transformações que ocorrem no planejamento didático referente ao processo de formação de habilidades em situações de ensino. Dessa maneira, essa teoria orienta o professor a realizar um teste diagnóstico inicial, que permita identificar o grau de desenvolvimento dessa habilidade. Para isso, é necessário identificar os conhecimentos específicos e indispensáveis, que antecedem os conhecimentos a serem aprendidos, e definir o domínio das ações cognoscitivas que se pretende construir. Supondo que se o estudante possui uma habilidade desenvolvida, isso implica que o aprendizado será uma atualização dessa habilidade. 


\section{Considerações Finais}

Portanto, compreende-se que as concepções da Teoria Histórico-Cultural de Vygotsky são complementadas na Teoria da Atividade de Leontiev, a qual se completa na Teoria da Assimilação de Galperin. E, com isso, o autor evidencia a importância do planejamento didático com enfoque na Teoria da Assimilação em articulação com as etapas da Atividade Histórico-Cultural para a prática docente e para realizar situações de aprendizagem que oportunizem a formação de conceitos e habilidades, de modo que haja o desenvolvimento integral da personalidade do aluno.

Este trabalho apresentou o itinerário formativo das teorias propostas por Vygotsky, Leontiev e Galperin pontuando, assim, em quais aspectos elas se complementam e divergem. Ademais, espera-se que este artigo sirva como um aporte teórico para pesquisas que buscam trabalhar na perspectiva teórica de Galperin. E pretende-se instigar o leitor a refletir na possibilidade de articular as concepções de Galperin com outras teorias socioculturais como, por exemplo, a Teoria da Objetivação de Luis Radford, a qual tem muitas implicações no ensino de Ciências e Matemática vislumbrando, dessa forma, sua extensão a outras áreas de conhecimento.

\section{Referências}

Bortolanza, A. M. E., \& Ringel, F. (2016) Vygotsky e as origens da teoria histórico-cultural: estudo teórico. Educativa, Goiânia, 19(1), 1020-1042.

Cenci, A., \& Damiani, M. F. (2018) Desenvolvimento da Teoria Histórico-Cultural da Atividade em três gerações: Vygotsky, Leontiev e Engeström. Roteiro, Joaçaba, 43(3), 919-948.

Cerezuela, C., \& Mori, N. N. R. (2015) A Educação Escolar e a Teoria Histórico-Cultural. XII Congresso Nacional de Educação - EDUCERE, $1251-1264$.

Cervo, A. L., Bervian, P. A., \& Silva, R. (2007) Metodologia Cientifica. (6a ed.), Pearson.

Leóntiev, A. N. (1978) Actividad, conciencia y personalidad. Ediciones Ciencias del Hombre.

Lobman, C. (2016) Três Dialéticas e Ambientes Sociais Terapêuticos de Aprendizagem. Organizadores: Maria Valéria Barbosa; Stela Miller; Suely Amaral Mello. Teoria histórico-cultural: questões fundamentais para a educação escolar. Marília: Oficina Universitária. São Paulo: Cultura Acadêmica, pp. $125-137$.

Mascarenhas, S. A. (2012) Metodologia Cientifica. Pearson, 128p.

Núñez, I. B. (2009) Vygotsky, Leontiev e Galperin: formação de conceitos e princípios didáticos. Liber livro. 216 p.

Núñez I. B. (1996) El proceso de asimilación en la perspectiva de la teoría de la actividad. Expressão. 27 (1); $41-52$.

Núñez I. B., \& Farias, T. C. L. (2004) O enfoque sócio-histórico-cultural da aprendizagem: os aportes de L.S. Vygotsky, A.N. Leontiev e Ya. Galperin. In: Nuñez, I. B., \& Ramalho, B. L. Fundamentos do ensino aprendizagem das ciências naturais e da matemática: o novo ensino médio. Sulina, pp. 51-68.

Núñez I. B., \& Gonzalez, O. P. (1997) La formación de conceptos científicos: una perspectiva desde la teoría de la actividad. EDUFRN.

Núñez I. B., \& González, P. O. (1998) Formação de conceitos segundo a Teoria da Assimilação de Galperin. Cadernos de Pesquisa, (105), 92-109.

Núñez, I. B., \& Ramalho, B. L. (2015) A teoria de P. Ya. Galperin como fundamento para a formação de habilidades gerais nas aulas de química. Redequim, $1(1), 5-19$.

Santos, M. A., \& Asbahr, F. S. F. (2020) A Teoria da Atividade de A. N. Leontiev: uma síntese a partir de suas principais obras. Revista Brasileira da Pesquisa Sócio-Histórico-Cultural e da Atividade, 2(2), 1-23. 